\title{
„Die Coronakrise zeigt uns die Lücken im System“
}

\author{
Marion Anna Becker
}

\section{Die Corona-Pandemie hat die Welt in einem Maße verändert, wie es vor einigen Monaten wohl kaum jemand für möglich gehalten hätte. DIE HEBAMME hat bei zwei Kolleginnen nachgefragt, wie sie die Krise meistern. Außerdem sprachen wir mit DHV-Präsidentin Ulrike Geppert-Orthofer über Herausforderungen, aber auch Chancen, die jetzt für die Berufsgruppe entstehen (Stand: 25.05.2020).}

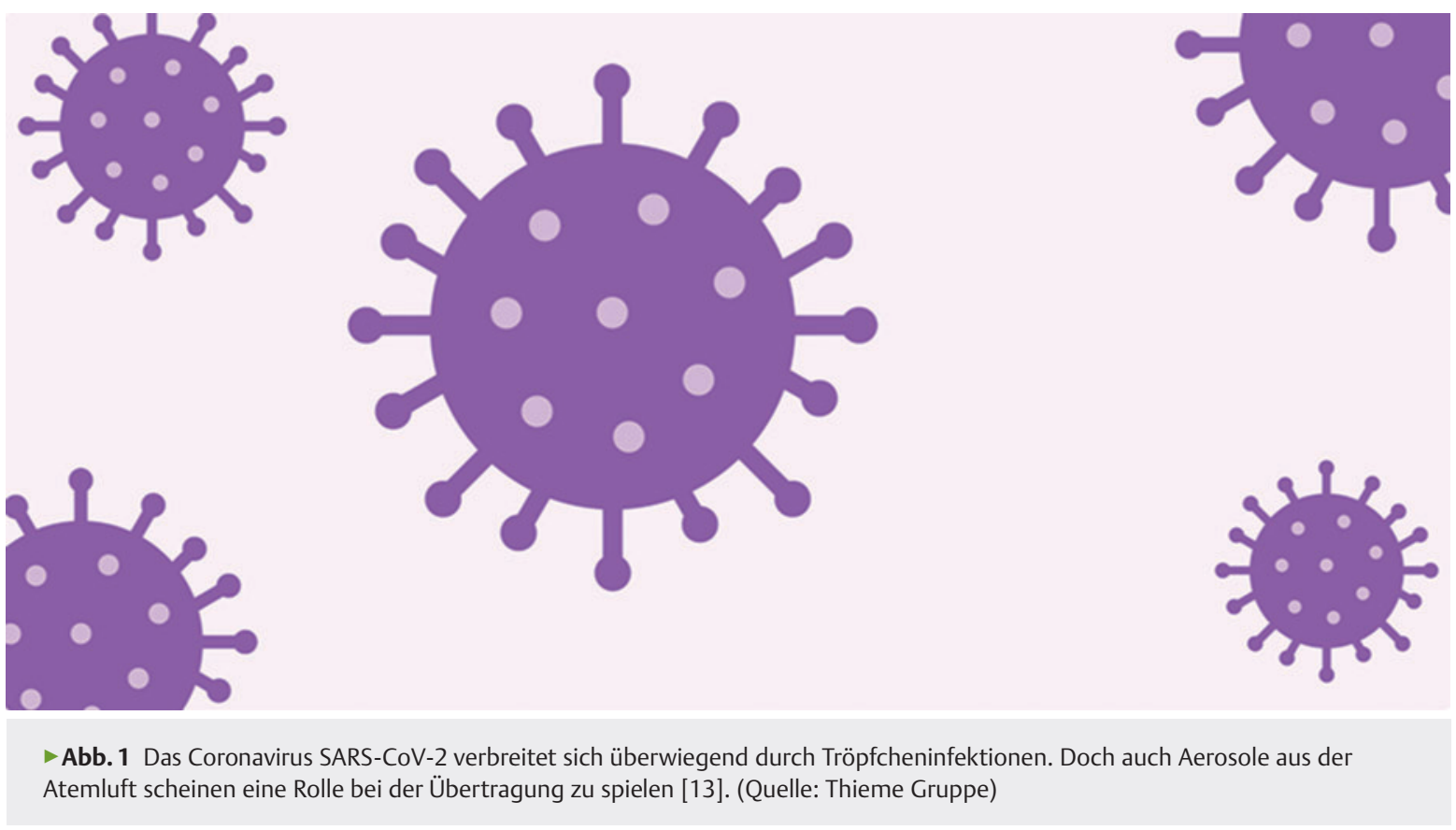

„In diesem Jahr ist alles anders“, sagte die Präsidentin des Deutschen Hebammenverbandes (DHV) in ihrer Videobotschaft zum Internationalen Hebammentag am 5. Mai 2020 [6]. Ein Jahr, in dem zahlreiche Feierlichkeiten geplant waren, um die Arbeit von Pflegefachpersonen zu würdigen. Es ist das Internationale Jahr der Pflegenden und Hebammen. Zwei Berufsgruppen, die durch die Corona-Pandemie wie viele Angehörige medizinischer Berufe vor ungeahnte Herausforderungen gestellt sind.

\section{Zeit der Ungewissheit: Der Beginn der Pandemie}

Rund vier Monate sind vergangen, seit mit einem Patienten aus Bayern der erste bestätigte Fall der Coronavirus-
Erkrankung (COVID-19) in Deutschland bekannt wurde [4]. Knapp einen Monat später, Mitte Februar 2020, mehrten sich Berichte über ein Infektions-Cluster im nordrhein-westfälischen Kreis Heinsberg. Auf einer Karnevalssitzung im Ort Gangelt hatte sich eine Reihe von Menschen mit dem Virus infiziert [15]. Der Landkreis wurde in der Folge zu einem Risikogebiet erklärt.

Als leitende Hebamme in den Städtischen Kliniken Mönchengladbach, nur knapp 40 Kilometer von Gangelt entfernt, war Claudia Moll unmittelbar mit dem Infektionsgeschehen in dem Risikogebiet konfrontiert. „Es fing an, dass Kolleginnen involviert waren, die aus dem Kreis Heinsberg kommen“, erinnert sich die 52-jährige. „Jeder kannte jemanden, der mit auf der Karnevalssitzung war, sodass große Unsicherheit herrschte und jeder sich gefragt hat: Könnte ich Teil dieser 
Infektionskette sein? Es hat eine Weile gedauert, bis wir wussten, wie wir mit dieser Entwicklung umgehen sollten.“

Diese Zeit der ersten Ungewissheit hat auch Daniela Degen erlebt. Sie ist freiberufliche Hebamme in Köln, wo es mit 225 Fällen pro 100000 Einwohnern, verglichen mit anderen Landkreisen, relativ viele bestätigte COVID-19Fälle gibt [12]. „Ich war erst mal vollkommen verunsichert und wusste nicht, wie ich überhaupt weiterarbeiten soll“, schildert sie ihre Gefühle von Anfang März. „Es war total unklar, wie man sich bei Wochenbettbesuchen verhalten sollte. Und es war kaum möglich, überhaupt an Informationen zu kommen.“

\section{Wie sich eine Klinik auf die Krise einstellt}

Zu Beginn der Pandemie beruhen alle Informationen über das neuartige Coronavirus (SARS-CoV-2) auf Daten, die in Zusammenhang mit dem ersten beschriebenen Ausbruch in der chinesischen Stadt Wuhan gesammelt wurden. Es sind nur wenige Fälle von Schwangeren und von Geburten dokumentiert. Doch durch die schnelle und weltweite Verbreitung des Virus wächst auch das Wissen rasant. Täglich kommen neue Erkenntnisse über Infektionswege, Krankheitsverlauf und Folgen von COVID-19 hinzu.

\section{UPDATE \\ COVID-19 \\ Häufigste Symptome}

Neben verbreiteten Symptomen wie Fieber, Husten und Halsschmerzen treten bei einem Großteil der Patienten Geruchs- und Geschmacksstörungen auf [13]. In einer Multicenter-Studie der Universität Mons in Belgien wurden 417 Patienten mit milden bis moderaten COVID-19-Symptomen aus 12 europäischen Krankenhäusern befragt. 85,6\% bzw. $88,0 \%$ der Teilnehmer gaben an, an olfaktorischer bzw. gustatorischer Dysfunktion zu leiden [9].

\section{Übertragungswege}

Als Hauptübertragungsweg von SARS-CoV-2 gilt weiterhin die Tröpfcheninfektion. Darüber hinaus wurden Coronavirus-RNA-haltige Aerosole in der Ausatemluft von Patienten oder in Patientenzimmern nachgewiesen. Da auch beim normalen Sprechen und Singen Aerosole freigesetzt werden, ist derzeit davon auszugehen, dass diese bei der Verbreitung von COVID-19 eine größere Rolle spielen könnten, als zunächst vermutet. Eine Übertragung über kontaminierte Oberflächen ist vor allem in der direkten Umgebung von infizierten Personen nicht auszuschließen. Daneben konnten PCR-positive Stuhl- und Konjunktivalproben nachgewiesen werden. Welche Bedeutung diese beiden Übertragungswege haben und ob es sich dabei um vermehrungsfähige Viren handelt, ist noch unklar.

Im medizinischen Bereich sollten alle potenziellen Übertragungswege in Betracht gezogen werden. Deshalb empfiehlt das RKI die Verwendung von persönlicher Schutzausrüstung (PSA) bestehend aus Schutzkittel, Einweghandschuhen, dicht anliegender Atemschutzmaske (FFP2 bzw. FFP3 oder Respirator bei ausgeprägter Exposition gegenüber Aerosolen, z. B. bei Intubation) und Schutzbrille [13].

\section{Inkubationszeit}

Die Zeit von der Ansteckung bis zum Symptombeginn liegt unverändert bei 1-14 Tagen (Median 5-6 Tage) [13].

\section{Infektiosität}

Ab wann ein Patient mit COVID-19 ansteckend ist, lässt sich noch nicht mit Sicherheit sagen. Bisherige Daten weisen darauf hin, dass bereits zwei Tage vor Symptombeginn eine relevante Infektiosität besteht und diese einen Tag vor Beginn der Symptome ihren Höhepunkt erreicht. Auch wie lange die Infektionsgefahr anhält, ist nicht abschließend geklärt. In ersten Untersuchungen enthielten Rachenabstriche bis zu vier Tage nach Symptombeginn vermehrungsfähige Viren, Sputumproben bis zu acht Tage [13].

\section{Krankheitsverlauf}

Durch die wachsende Anzahl an Daten lässt sich mittlerweile gut erkennen, dass es keinen typischen Verlauf von COVID-19 gibt: Die Erkrankung variiert von Patient zu Patient und kann auch asymptomatisch bleiben [13]. So ergab z. B. die viel diskutierte „Heinsberg-Studie“ mit 919 Teilnehmern bei $22 \%$ der positiv getesteten Probanden einen asymptomatischen Verlauf [15]. Am häufigsten scheinen jedoch Verläufe mit milder bis moderater Ausprägung zu sein. Ein schwerer Krankheitsverlauf mit Lungenversagen oder Tod ist seltener, wird aber auch bei Personen ohne Vorerkrankung und bei jüngeren Menschen beobachtet [13].

\section{Risikogruppen}

Als Risikogruppen, die besonders häufig von schweren Verläufen betroffen sind, gelten weiterhin Personen ab 50-60 Jahre und Personen mit

Vorerkrankungen der Lunge / des Herzens, mit chronischen Lebererkrankungen, Diabetes mellitus, Krebserkrankungen und geschwächtem Immunsystem. Auch stark adipöse Menschen und Raucher sind gefährdet [13].

\section{Medikation und Impfung}

Bisher steht weder eine Medikation noch eine Impfung gegen COVID-19 zur Verfügung. Es gibt jedoch eine ganze Reihe potenzieller Impfstoffe. Zehn davon werden aktuell in klinischen Studien untersucht [13]. 


\section{Immunität}

Nach einer Infektion mit SARS-CoV-2 scheinen die Patienten spezifische Antikörper gegen das $\mathrm{N}$ - und das S-Protein des Coronavirus zu entwickeln. Diese sind im Durchschnitt nach 5-12 Tagen (IgM) bzw. nach 14 Tagen (lgG) im Blut nachweisbar. Wie stabil die Immunität ist und wie lang sie andauert, wird jedoch erst durch serologische Längsschnittuntersuchungen geklärt werden können [13]

Claudia Moll berichtet, dass der Kreißsaal der Städtischen Kliniken Mönchengladbach sich rasch auf die neue Situation eingestellt hat: „Zuerst beschränkten sich Maßnahmen wie die Erfassung von Risikofaktoren mithilfe eines Fragebogens auf Frauen aus dem Risikogebiet Heinsberg. Dann wurde relativ schnell ein Fragebogen entwickelt, den jede Frau ausfüllen muss, bevor sie den Kreißsaal betritt." Darin gehe es um Fragen, wie: Hatten Sie schon mal einen positiven Abstrich? Hatten Sie in letzter Zeit Fieber? Hatten Sie Kontakt zu Personen mit bestätigter Infektion? Insgesamt müssten die Frauen zehn Fragen beantworten. Je nachdem, welche mit „Ja“ beantwortet würden, fände dann eine Risikoeinschätzung statt. „Auf diese Weise können Frauen mit Verdacht auf COVID-19 schnell identifiziert und separiert werden“, erklärt Moll.

Auch für bestätigte COVID-19-Fälle und für Verdachtsfälle entwickeln die Städtischen Kliniken Mönchengladbach ein methodisches Vorgehen. Claudia Moll berichtet, dass für die ambulante Betreuung der betroffenen Frauen ein separater Bereich vorgesehen sei, in dem Ultraschalluntersuchungen oder Blutabnahmen stattfinden könnten. Um mögliche Infektionsketten einzuschränken, sei als einzige Kontaktperson eine speziell geschulte Oberärztin für die Betreuung der Frauen vorgesehen. Für die Geburt selbst stehe ein genauer Ablaufplan zur Verfügung, an den sich betroffene Frauen streng halten müssten. Moll: „Der Plan sieht vor, dass die Frau in der Klinik anruft und ihr Kommen ankündigt, sobald sie Wehen verspürt. Wenn sie das Gelände betritt, muss sie sich erneut melden, um an einem speziellen Hintereingang in Empfang genommen zu werden. In der Zwischenzeit kann sich das Kreißsaalpersonal umziehen. Die Frau wird dann in einen Isolationskreißsaal geleitet, den wir speziell für Frauen mit bestätigter Infektion oder mit ausstehendem Testergebnis vorgesehen haben.“

Claudia Moll berichtet, dass für die betroffenen Frauen die kontinuierliche Betreuung durch eine Hebamme vorgesehen sei, da der Isolationskreißsaal von so wenig Personal wie möglich betreten werden dürfe. Die Frau verbleibe die gesamte Geburt über in dem Kreißsaal. Wenn sie ihr Kind nach der Geburt bonden wolle, sehe eine Standardanweisung vor, dass sie bestimmte Hygieneregeln einhalten müsse. Dazu gehöre, dass sie die 
Oberflächen mit Seifenlauge abwaschen und einen Mund-Nasen-Schutz tragen müsse. Moll erklärt: „Sie bleibt nach der Geburt zwei Stunden in diesem Kreißsaal und wird dann auf die Wöchnerinnenstation verlegt. Hier ist wieder ein separiertes Zimmer für sie vorgesehen, in dem sie die gesamte Zeit über mit ihrem Kind bleibt. Alle Untersuchungen, wie beispielsweise die U2 durch den Kinderarzt, finden in diesem Zimmer statt.“

\section{WEB-TIPP}

Mehr Informationen zur getrennten Versorgung von COVID-19-Fällen, Verdachtsfällen und anderen Patienten im stationären Bereich stellt das Robert Koch-Institut zur Verfügung unter: https: / / www.rki.de/DE/Content/InfAZ/N/Neuartiges_Corona virus/Getrennte_Patientenversorg_stationaer.html

\section{Neue Normalität: Wochenbettbesuche auf Abstand}

Auch Daniela Degen passt ihre Besuche bei den Familien an die neuen Gegebenheiten an. Dabei hilft ihr in den ersten Tagen der Corona-Pandemie eine FacebookGruppe, in der sie sich mit anderen Hebammen zu den drängendsten Fragen austauscht. „Kurz darauf ist auch der Informationsfluss vom DHV angelaufen“, berichtet sie. „Wir haben ganz klare Verhaltensregeln bekommen, was wir bei Wochenbettbesuchen beachten müssen.“ Dazu zählt z. B. die Empfehlung, Besuchskontakte generell auf ein Minimum zu reduzieren und zu prüfen, ob eine Beratung per Video oder Telefon möglich und sinnvoll ist. Degen handhabt es so: „Termine, die wirklich wichtig sind, wie die ersten Tage nach der Geburt, versuche ich weiterhin real vorzunehmen. Wenn es aber im weiteren Verlauf darum geht, Fragen zu klären, für die nicht unbedingt der persönliche Kontakt nötig ist, ermögliche ich diese durch Videotelefonie oder durch ein normales Telefonat. So kann ich trotzdem für die Familie da sein, aber eben virtuell.“

Auch für ihre Kurse zur Geburtsvorbereitung und Babymassage, die Degen in der Elternschule „Neue Kölner e. V. “ anbietet, muss sie sich komplett umstellen. Mitten in einem laufenden Wochenendkurs erhält sie die Nachricht vom lokalen Gesundheitsamt, dass die Elternschule zum Schutz der weiteren Ausbreitung von COVID-19 geschlossen werden muss. „Ich fand es furchtbar, die Eltern so im Regen stehen zu lassen“, berichtet die 45Jährige. „Zuerst hatte ich auch gar nicht das technische Equipment, wie Mikrofon und Kamera, um meine Kurse online fortzuführen. Auch meine Internetverbindung hat nicht gereicht. Es hat knapp drei Wochen gedauert, bis ich diese Stolpersteine überwunden hatte." Degen berichtet, dass sie und ihre Kolleginnen viele Tage und
Stunden investiert hätten, um neue Konzepte zu erarbeiten, damit die Kurse der Elternschule auch online weiterlaufen konnten.

\section{Nähe lässt sich nur bedingt ersetzen}

Für Hebammen ist es ein großer Vorteil, dass sie bestimmte Beratungen und Kurse für die Zeit der Coronakrise auch telefonisch oder online durchführen können und dass diese, gemäß der Sonderregelungen, die der DHV erwirkt hat, nun auch abrechenbar sind. Alle Probleme, die durch die Kontaktbeschränkungen entstehen, lassen sich dadurch allerdings nicht lösen. „Die Kolleginnen berichten teilweise von erheblichen Einkommenseinbußen, weil viele Termine nicht mehr wahrgenommen werden“, sagt DHV-Vorsitzende Ulrike Geppert-Orthofer. „Leider können auch die Sonderregelungen diesen Nachfragerückgang nicht ausgleichen. Das zeigt, dass Hebammenarbeit eine sehr intime Tätigkeit ist, die Nähe erfordert. Und da greifen virtuelle Lösungen eben nur bedingt."

Diese Erfahrung macht auch Daniela Degen. Ihr fehlt die Nahbarkeit, das Zwischenmenschliche, das ihr Beruf normalerweise mit sich bringt. Dazu gehöre, Wöchnerinnen mal in den Arm zu nehmen, wenn es ihnen nicht gut gehe oder das Baby zu halten, wenn die Mutter kurz etwas zu Hause erledigen müsse. „Das sind Dinge, die ich im Moment vermeide“, erklärt die Hebamme. „Ich versuche, so wenig Körperkontakt wie möglich mit den Familien aufzunehmen. Auch wenn ich mir die Kinder anschaue, mache ich das immer erst mal mit Abstand, um wirklich nur das Allernötigste am Baby selbst zu verrichten." Dadurch fühle sie sich allerdings sehr gehemmt und habe immer ein bisschen das Gefühl, ein Eindringling zu sein, der in das Nest Familie komme und Überträger des Virus sein könne, sagt Degen.

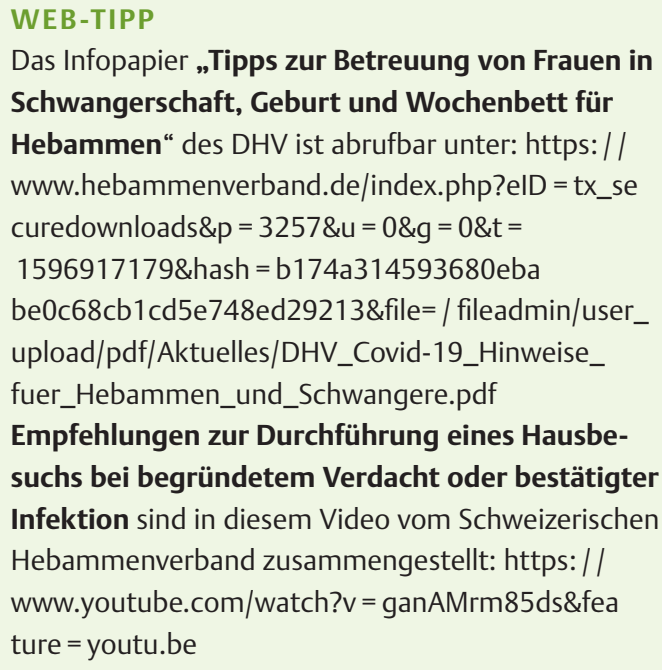




\section{Die Lücken im System}

Auch Kreißsaalleiterin Claudia Moll schildert von sich und ihren Kolleginnen die Sorge, potenziell Überträgerin von SARS-CoV-2 zu sein: „Die Angst, sich anzustecken ist schon da. Dabei geht es aber zunächst gar nicht um die eigene Gesundheit, sondern um die Befürchtung, Kolleginnen oder Risikopersonen in der Familie anzustecken.“ Die Nähe, die ihr Beruf erfordert, ist für Moll und ihre Kolleginnen normalerweise ganz selbstverständlich. Doch in der jetzigen Situation, so sagt sie, mache man sich den körperlichen Kontakt zu den Gebärenden manchmal sehr viel mehr bewusst als früher.

Beide Hebammen geben an, dass sie derzeit gut mit Schutzkleidung ausgestattet seien. Auch wenn sie zwischendurch erfinderisch werden mussten und sich - wie Claudia Moll und ihr Team - z. B. Visiere selbst gebastelt haben.

Optimal ist die Versorgung mit Schutzausrüstung jedoch weiterhin nicht, wie Geppert-Orthofer sagt. „Es gibt immer noch Kolleginnen in Kliniken, die uns berichten, dass sie den gesamten Acht-Stunden-Dienst über den gleichen Mund-Nasen-Schutz verwenden müssen. Und auch einigen freiberuflichen Hebammen mangelt es weiterhin an Schutzkleidung." Die DHV-Präsidentin beklagt, dass es noch keine einheitliche Regelung für die Verteilung von Schutzausrüstung an Hebammen gäbe und dass die Kolleginnen in diesem Punkt sehr auf sich allein gestellt seien. Doch zumindest für die Kosten, die Hebammen für Schutzausrüstung aufbringen müssen, konnte der Verband eine befristete Regelung mit den gesetzlichen Krankenkassen aushandeln (s. Kasten).

\section{INFO}

Materialpauschalen für freiberufliche Hebammen Am 05.05.2020 verkündete der DHV auf seiner Webseite, dass er Materialpauschalen für persönliche Schutzausrüsten mit dem GKV-Spitzenverband vereinbaren konnte. Die Regelung gilt für erbrachte Leistungen im Zeitraum vom 19.03.2020 bis 19.06.2020. Mehr Informationen unter: https: / / www.hebammenverband.de/aktuell/nachricht-detail/ datum/2020/05/05/artikel/zuschlaege-fuer-denmehraufwand-von-schutzausruestung/

Auch in vielen anderen Bereichen kämpft der Verband weiter für die Rechte der Hebammen. „Die Coronakrise zeigt uns ganz deutlich die Lücken im System“, sagt Geppert-Orthofer. „Wir nehmen wahr, dass überhaupt nicht erkannt wurde, wie wichtig die Zeit der Familienbildung für die Gesundheit der Familie und für Mutter und Kind ist und damit letztlich für die Grundlage unserer Gesellschaft. “ Anders ist es für sie nicht zu erklären, dass im Zuge von Besuchsbeschränkungen für kranke und 
pflegebedürftige Menschen in manchen Kliniken auch keine Begleitperson mehr im Kreißsaal zugelassen wurde. Eine Einschränkung, die bei vielen werdenden Müttern zu heller Aufregung und letztlich zu einem Ansturm auf Geburtskliniken geführt hat, bei denen das Kreißsaalverbot für den Partner nicht galt. Auch der DHV und die Deutsche Gesellschaft für Gynäkologie und Geburtshilfe (DGGG) haben sich ausdrücklich dafür ausgesprochen, auch weiterhin eine Begleitperson für die Geburt zuzulassen [3][5]. Die meisten Kliniken haben die Beschränkungen daraufhin wieder aufgehoben.

An anderen Stellen sind jedoch noch keine Verbesserungen in Sicht. „Hebammen werden bei vielen Verordnungen schlichtweg vergessen!“, kritisiert Geppert-Orthofer. So erhielten die Kolleginnen immer noch nicht in allen Bundesländern einen Platz für die Kindernotbetreuung. Außerdem fehle ein Rettungsschirm für freiberufliche Hebammen, der Einkommenseinbußen durch die Corona-Pandemie auffangen könne. Und natürlich gehe es darum, Fachkräfte aus dem Gesundheits- und Pflegebereich generell besser zu bezahlen und sie stärker in Entscheidungen einzubinden. Die DHV-Präsidentin zieht daraus folgenden Schluss: „Aus meiner Sicht ist das der entscheidende Punkt, den wir aus der Krise lernen müssen - dass wir aktuell ein Wertesystem haben, das nicht widerspiegelt, welche Berufe unser Überleben und unsere Gesundheit sichern oder fördern. Das ist natürlich keine Aufgabe, die der Hebammenverband allein bewältigen kann. In meinen Augen wird das die große gesellschaftliche Herausforderung: es zu schaffen, nicht zur alten Normalität zurückzukehren.“

\section{HINTERGRUNDWISSEN \\ Positives Geburtserlebnis - auch in Zeiten der Corona-Pandemie}

In einer Pressemitteilung vom 18.05.2020 nimmt der DHV Stellung zur Maskenpflicht im Kreißsaal für

Gebärende, die in erster Linie das Infektionsrisiko von Hebammen, Ärztinnen und Ärzten reduzieren soll. Der Verband weist darauf hin, dass der Mund-NasenSchutz Frauen daran hindern könne, frei zu atmen, was jedoch zur Schmerzerleichterung und Verarbeitung der Wehen äußerst wichtig sei. Deshalb spricht er sich dafür aus, Abstriche bei den Frauen durchzuführen, damit ihr Infektionsstatus bei oder kurz nach der Aufnahme in die geburtshilfliche Abteilung relativ sicher bekannt sei. Und es müsse gewährleistet sein, dass ausreichend Schutzausrüstung für medizinisches Personal zur Verfügung stehe. Würden Hebammen, Ärztinnen und Ärzte eine medizinische Maske tragen, erübrige sich der Mund-Nasen-Schutz für die Gebärende, begründet der DHV.

Auch die WHO erinnert mit einprägsamen Infografiken daran, dass Frauen ein Recht auf ein positives Geburtserlebnis haben, ganz gleich ob mit bestätigter SARS-CoV-2-Infektion oder ohne. Mehr Infos unter: https://www.who.int/reproductivehealth/publications/emergencies/COVID-19pregnancy-ipc-breastfeeding-infographics/en/

\section{Warum die Krise auch Chancen bietet}

Ulrike Geppert-Orthofer betont auch, dass es gerade während der Corona-Pandemie wichtiger denn je sei, Schwangere, Mütter und Familien bestmöglich zu betreuen. Hebammen begleiten Frauen in einer der entscheidendsten Lebensphasen und haben dadurch auch die Möglichkeit, frühzeitig auf Sorgen und Nöte der Frauen aufmerksam zu werden. Die ungewisse Entwicklung der Coronakrise kann in der Zeit der Schwangerschaft als besonders bedrohlich erlebt werden. Neben der Angst vor der Viruserkrankung für die eigene Gesundheit und die des Babys können die Eltern durch finanzielle Unsicherheiten und durch die fehlende familiäre Unterstützung z. B. durch Großeltern belastet sein [14].

Doch es lässt sich auch Positives berichten. Denn eine Zeit, in der alles auf den Kopf gestellt ist, bietet auch die Möglichkeit, viele Gewohnheiten zu hinterfragen. So hat Daniela Degen die Kontaktbeschränkungen in der Zeit des Lockdowns für ihre Wochenbettbesuche als überaus positiv wahrgenommen: „Die Eltern mussten sich nicht mehr rechtfertigen, dass sie noch keinen Besuch haben wollten. Es war einfach klar, dass niemand kommen konnte. Dadurch habe ich in den Familien eine unglaubliche Ruhe und Friedlichkeit erlebt. Das war wirklich schön für das Baby und für die frischgebackenen Eltern.“

Claudia Moll macht im Kreißsaal in Mönchengladbach ähnliche Erfahrungen: „Man merkt deutlich, dass weniger Publikumsverkehr herrscht. Und da im Kreißsaal auch nur eine Person dabei sein darf und kein Wechsel stattfindet, haben wir das Gefühl, viel mehr wahrzunehmen und einen engeren Bezug zur Begleitperson und natürlich zu der Gebärenden aufbauen können." Und sie erzählt, dass die Schwestern von der Wochenbettstation viel seltener mit Stillproblemen konfrontiert seien. Moll und ihre Kolleginnen führen das darauf zurück, dass die Frauen weniger Ablenkung durch Besuche hätten und sich stärker ihrem Baby widmen könnten. Daniela Degen sieht das genauso, denn auch bei ihren Wochenbettbesuchen in Köln kommt es während der Kontaktbeschränkungen kaum zu Stillproblemen. „Die Frauen glauben, ständig mit ihrem Umfeld in Austausch sein zu müssen“, schließt die Hebamme daraus, „dadurch sind sie manchmal abgelenkt und legen das Baby nicht direkt an, wenn die ersten Hungerzeichen kommen. Aktuell stelle ich das kaum noch fest, weil die Frauen sich wirklich auf ihre kleine Familie konzentrieren können.“ 
UPDATE

Relevanz von SARS-CoV-2 für Schwangerschaft und Geburt

Insgesamt ist die Datenlage zu Schwangerschaften und Geburten SARS-CoV-2-positiver Mütter weiterhin gering, sodass alle Ergebnisse zum jetzigen Zeitpunkt vorsichtig interpretiert werden müssen. Schwangere mit COVID-19 scheinen vor allem milde bis moderate Symptome zu entwickeln, auch asymptomatische Verläufe sind zu beobachten. In einer Fallserie aus New York wurden innerhalb von zwei Wochen 215 Frauen, die zur Geburt in das Krankenhaus kamen, auf eine SARS-CoV-2-Infektion getestet. 15,4\% der nasopharyngealen Abstriche waren positiv. 13,5\% der infizierten Frauen waren asymptomatisch. Nur vier Frauen zeigten bei ihrer Aufnahme Symptome wie Husten, Fieber oder Kurzatmigkeit, drei weitere entwickelten Symptome während ihres Klinikaufenthalts [14][16]. Ob Schwangere ein höheres Risiko für einen schweren Verlauf von COVID-19 haben als nichtschwangere Frauen, lässt sich noch nicht eindeutig sagen. Die meisten Studien deuten bisher nicht darauf hin: An einer multizentrischen Studie des UK Obstetric Surveillance System (UKOSS) nahmen 16749 Patienten teil, die aufgrund einer Infektion mit SARS-CoV-2 stationär behandelt werden mussten. Darunter 427 Schwangere (6\%), von denen 40 (9\%) intensivmedizinisch betreut werden mussten. Der Anteil war vergleichbar mit nicht-schwangeren Frauen der gleichen Altersgruppe. Auch eine höhere Sterblichkeit konnte nicht ermittelt werden [14] [11]. Zwei Studien mit kleinen Fallzahlen kommen dagegen zu dem Ergebnis, dass schwangere Frauen mit COVID-19 signifikant häufiger zu schweren oder kritischen Krankheitsverläufen neigen als nichtschwangere Frauen [2][19].

Ob durch COVID-19 ein erhöhtes Risiko für Fehlgeburten besteht, ist noch unklar. Bisher werden nur Einzelfälle beschrieben, bei denen SARS-CoV-2-positive Frauen eine Fehlgeburt erlitten [10][1]. In einem Fallbericht konnte in diesem Zusammenhang eine Infektion der Plazenta festgestellt werden [1]. Entscheidend für den Krankheitsverlauf scheint der Infektionszeitpunkt zu sein: In der UKOSS-Studie wurden die meisten Frauen vor allem im späten zweiten und dritten Trimester hospitalisiert. Das stimmt mit den Erkenntnissen zu anderen respiratorischen Viruserkrankungen überein, bei denen Frauen in der späten Schwangerschaft zu schwereren Verläufen neigten. Schwangere sollten sich daher insbesondere in der fortgeschrittenen Schwangerschaft durch strikte Einhaltung von Abstands- und Hygieneregeln vor einer Ansteckung schützen [10].
Für eine vertikale Übertragung von SARS-CoV-2 von der Mutter auf das Baby (prä- oder intrapartal) gibt es nur sehr wenige Hinweise. In zwei Fallberichten gelang der Nachweis von IgM im neonatalen Serum. Da IgM nicht plazentagängig ist, könnte das eine Infektion in utero beweisen [8][20]. Aufgrund der kleinen Fallzahlen und weiterer Limitationen sind die Ergebnisse jedoch vorsichtig zu bewerten.

Zudem zeigt die Mehrzahl der Neugeborenen von SARS-CoV-2-positiven Mütter keine Krankheitszeichen [14][13].

Ein Nachweis von SARS-CoV-2 in der Muttermilch gelang bisher nicht. Daher ist davon auszugehen, dass die Übertragung des Virus von der Mutter auf das Baby vor allem durch eine Tröpfcheninfektion nach der Geburt stattfand [14][13].

Verschiedene Studien legen nahe, dass COVID-19 mit einer Hyperkoagulabilität einhergeht [18][17]. Da es in der Schwangerschaft physiologisch zu einer verstärkten Blutgerinnung kommt, ist es wahrscheinlich, dass das Risiko für maternale Venenthrombosen durch eine Infektion mit SARS-CoV-2 zusätzlich erhöht ist. Verringerte Mobilität durch Quarantäne oder Krankenhausaufenthalte sind weitere Risikofaktoren [14]. Deshalb sollte bei Frauen mit bestätigter Infektion oder mit Verdacht auf COVID-19 eine Prophylaxe für venöse Thromboembolien (VTE) mit niedermolekularem Heparin erwogen werden [13][14].

\section{Autorinnen / Autoren}

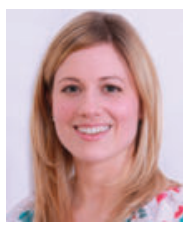

Marion Anna Becker ist freie Journalistin aus Bad Homburg und hat sich auf Gesundheitsthemen spezialisiert.

DIE HEBAMME bedankt sich ganz herzlich bei Daniela Degen, Claudia Moll und Ulrike Geppert-Orthofer für die Interviews, die unsere Autorin im April und Mai 2020 mit ihnen führen durfte.

Korrespondenzadresse

Marion Anna Becker

Höhestraße 34

61348 Bad Homburg

E-Mail: m.becker@gesund-informiert.de

Literatur

[1] Baud D, Greub G, Favre G et al. Second-Trimester Miscarriage in a Pregnant Woman With SARS-CoV-2 Infection [Published online April 30, 2020]. JAMA 2020, doi:10.1001/ jama.2020.7233 
[2] Collin J, Bystrom E, Carnahan A, Ahrne M. Pregnant and postpartum women with SARS-CoV-2 infection in intensive care in Sweden [published online ahead of print, 2020 May 9]. Acta obstetricia et gynecologica Scandinavica 2020 , doi:10.1111/aogs.13901

[3] Deutsche Gesellschaft für Gynäkologie und Geburtshilfe (DGGG). DGGG empfiehlt: Väter bei der Geburt zulassen Auch in Zeiten der Corona-Pandemie (18.05.2020). Im Internet: https://www.dggg.de/presse-news/pressemitteilungen/ mitteilung/dggg-empfiehlt-vaeter-bei-der-geburt-zulassen-a uch-in-zeiten-der-corona-pandemie-1195/; Stand: 26.03.2020

[4] Deutsche Presseagentur (dpa). Erster Coronafall in Deutschland bestätigt (21.05.2020). Im Internet: https://www.sued deutsche.de/gesundheit/krankheiten-erster-coronavirus-fallin-deutschland-bestaetigt-dpa.urn-newsml-dpa-com-200901 01-200128-99-659450; Stand: 28.01.2020

[5] Deutscher Hebammenverband (DHV). Gebären in CoronaZeiten: Frauen nicht allein lassen (18.05.2020). Im Internet: https: / /www.hebammenverband.de/aktuell/nachricht-detail/ datum/2020/04/17/artikel/gebaeren-in-corona-zeiten-frauennicht-alleine-lassen/; Stand: 17.04.2020

[6] Deutscher Hebammenverband (DHV): Grußbotschaft zum Internationalen Hebammentag 2020. (18.05.2020): Im Internet: https: / /youtu.be/2IAfTDKsXmM; Stand: 05.05.2020

[7] Deutscher Hebammenverband (DHV): Änderungsvereinbarung vom 25.03.2020 (21.05.2020). Im Internet: https: / / www.hebammenverband.de/index.php?elD $=$ tx_securedown loads \&p $=3257 \& u=0 \& g=0 \& t=1597680547 \&$ hash $=7$ a76bb617f2f017373819f9bc9204f2f6784be80\&file= / filead min/user_upload/pdf/Aktuelles/DHV_Aenderungsvereinba rung_Sonderregelungen_Corona.pdf; Stand: 25.03.2020

[8] Dong L, Tian J, He $S$ et al. Possible Vertical Transmission of SARS-CoV-2 From an Infected Mother to Her Newborn [published online ahead of print, 2020 Mar 26]. JAMA 2020, doi: $10.1001 /$ jama.2020.4621

[9] Lechien JR, Chiesa-Estomba CM, De Siati DR et al. Olfactory and gustatory dysfunctions as a clinical presentation of mildto-moderate forms of the coronavirus disease (COVID-19): a multicenter European study [published online ahead of print, 2020 Apr 6]. Eur Arch Otorhinolaryngol 2020; 1-11, doi:10.1007/s00405-020-05965-1

[10] Liu Y, Chen H, Tang K et al. Clinical manifestations and outcome of SARS-CoV-2 infection during pregnancy [published online ahead of print, $2020 \mathrm{Mar}$ 4]. J Infect. 2020, doi:10.1016/j.jinf.2020.02.028

[11] Knight Marian, Bunch Kathryn, Nicola Vousde et al. Characteristics and outcomes of pregnant women hospitalised with confirmed SARS-CoV-2 infection in the UK: a national cohort study using the UK Obstetric Surveillance System (UKOSS). medRxiv 2020, doi: https: / /doi.org/10.1101/2020.05.08.20089268
[12] Robert Koch-Institut (RKI). Robert Koch-Institut: COVID-19Dashboard (25.05.2020). Im Internet: https: / /experience.arc gis.com/experience/478220a4c454480e823b17327b2bf1d4/ page/page_1/; Stand: 18.05 .2020

[13] Robert Koch-Institut (RKI). SARS-CoV-2 Steckbrief zur Coronavirus-Krankheit-2019 (COVID-19) (25.05.2020). Im Internet: https: / /www.rki.de/DE/Content/InfAZ/N/Neuartiges_Corona virus/Steckbrief.html\#doc13776792bodyText1; Stand: 15.05.2020

[14] Royal College of Obstetricians and Gynaecologists (RCOG). Coronavirus (COVID-19) Infection in Pregnancy. Information for healthcare professionals - Version 9 (25.05.2020) https: / /www.rcog.org.uk/globalassets/documents/guidelines/202005-13-coronavirus-covid-19-infection-in-pregnancy.pdf; Stand: 13.05 .2020

[15] Streek H, Schulte B, Kümmerer B et al. Infection fatality rate of SARS-CoV-2 infection in a German community with a super-spreading event. medRxiv 2020; doi:https://doi.org/ 10.1101/2020.05.04.20090076

[16] Sutton D, Fuchs K, D'Alton $M$ et al. Universal Screening for SARS-CoV-2 in Women Admitted for Delivery [published online ahead of print, 2020 Apr 13]. N Engl J Med 2020, doi:10.1056/NEJMc2009316

[17] Thomas W, Varley A, Johnston E. Thrombotic complications of patients admitted to intensive care with COVID-19 at a teaching hospital in the United Kingdom [published online ahead of print, 2020 Apr 25]. Thrombosis Research 2020; 191: 7677, doi: 10.1016/j.thromres.2020.04.028

[18] Wichmann D, Sperhake JP, Lütgehetmann M et al. Autopsy Findings and Venous Thromboembolism in Patients With COVID-19: A Prospective Cohort Study [published online ahead of print, 2020 May 6]. Ann Intern Med. 2020, doi: $10.7326 / \mathrm{M} 20-2003$

[19] Yin M, Zhang L, Deng G et al. Severe Acute Respiratory Syndrome Coronavirus 2 (SARS-CoV-2) Infection During Pregnancy In China: A Retrospective Cohort Study. MedRxiv. 2020.

[20] Zeng $\mathrm{H}, \mathrm{Xu} \mathrm{C}$, Fan J et al. Antibodies in Infants Born to Mothers With COVID-19 Pneumonia [published online ahead of print, $2020 \mathrm{Mar}$ 26]. JAMA 2020, doi: 10.1001/ jama.2020.4861

\section{Bibliografie}

DOI https://doi.org/10.1055/a-1159-4797

Die Hebamme 2020; 33: 21-28

(c) Georg Thieme Verlag KG Stuttgart · New York ISSN 0932-8122 